\title{
El staff de prácticas profesionales en Trabajo Social: espacio de fomento del aprendizaje autodirigido. Un estudio de caso en la Universidad Católica Luis Amigó, Colombia
}

\section{Cristian Eduardo Blanco-García}

Magíster en Estudios Políticos. Comunicador Social-Periodista

Universidad Católica Luis Amigó. Medellín, Colombia

https:/ / orcid.org/0000-0003-2530-4409・cristian.blancoga@amigo.edu.co

Resumen

Las prácticas profesionales son un componente esencial de la formación profesional de los trabajadores sociales, en la consolidación del currículo y en el establecimiento de las relaciones entre el programa, la universidad y el contexto. Mientras que otros estudios se han centrado en los programas presenciales, este texto profundiza en el rol del staff de prácticas como espacio de refuerzo del aprendizaje autodirigido de los practicantes en los programas de educación a distancia. El argumento central de este artículo es que el aprendizaje autodirigido que requieren las prácticas profesionales exige no solo un aprender a aprender, sino también un aprender a hacer desde la recuperación selectiva de saberes previos. El análisis de los resultados muestra que tal recuperación no es sencilla para los estudiantes, por lo que se requieren estrategias adicionales en la supervisión de las prácticas para la obtención de mejores resultados. De otro lado, esta investigación expone que la modalidad distancia no supone un obstáculo para estos aprendizajes.

Palabras clave: Aprendizaje autodirigido; Trabajo Social; Prácticas profesionales; Supervisión de práctica.

Recibido: 12/11/2020 | Aprobado: 20/03/2021 |Publicado: 01/07/2021

(1)(2) Esta obra está bajo una Licencia Creative Commons Atribución-NoComercialCompartirIgual 4.0 Internacional.

Financiación o proveniencia del artículo: Este artículo es producto de la investigación Preparación para la práctica profesional: el staff de prácticas como espacio de aprendizaje en el programa de Trabajo Social modalidad distancia, realizada entre febrero y octubre de 2020. Esta investigación fue financiada a través de la convocatoria interna de la Universidad Católica Luis Amigó, Colombia de 2019.

¿Cómo citar este artículo? / How to quote this article?

Blanco-García, C. E. (2021). El staff de prácticas profesionales en Trabajo Social: espacio de fomento del aprendizaje autodirigido. Un estudio de caso en la Universidad Católica Luis Amigó, Colombia. Prospectiva. Revista de Trabajo Social e intervención social, (32), 259-274. doi: 10.25100/prts.v0i32.10750. 
Blanco-García

\title{
The staff of professional practices in Social Work: space for the promotion of self-directed learning. A case study at the Luis Amigó Catholic University, Colombia
}

\begin{abstract}
Internships are an essential component of the training of social workers. While other studies have focused on face-to-face programs, this text delves into the role of the internship staff as a space to reinforce the self-directed learning of practitioners in remote education programs. The central argument of this article is that the self-directed learning that professional practices require demand not only learning to learn, but also learning to do from the selective recovery of previous knowledge. The analysis of the results shows that such recovery is not easy for students, hence additional strategies are required in the supervision of the internships to obtain better results. On the other hand, this research shows that the distance modality does not represent an obstacle for such learning.
\end{abstract}

Keywords: Self-directed learning; Social Work; Internships; Supervision.

Sumario: 1. Introducción, 2. Metodología, 3. Hallazgos, 3.1 Percepciones sobre el alistamiento y preparación para las prácticas profesionales, 3.2 Utilidad percibida de las actividades de aprendizaje en el staff de prácticas, 3.3 Aprendizaje autodirigido, 4. Conclusiones, 5. Referencias bibliográficas. 


\section{Introducción}

Las prácticas profesionales son un componente esencial de la formación de los trabajadores sociales. Carmina Puig-Cruells (2020) define las prácticas en Trabajo Social como "el conjunto de actividades y funciones propias de los trabajadores sociales, que se realizan tanto en los marcos de las instituciones colaboradoras como aquellas que se realizan en el marco universitario" (p. 61). Estas actividades se realizan "con la intencionalidad de transformar situaciones problemáticas en función de demandas concretas por sujetos diversos" (Parola, 2020). En ellas los estudiantes aplican sus conocimientos y habilidades en agencias y contextos organizacionales y/o comunitarios. Esto es, los estudiantes piensan la relación teoría-práctica, examinan, critican y ponen a prueba sus conocimientos, valores y principios aprehendidos en los cursos académicos. En ese proceso, debe considerar y verse inmerso tanto en la investigación como en la intervención (García-Barreto, ZambranoCárdenas y Martínez-Suárez, 2017).

En el caso del programa de Trabajo Social, modalidad distancia, de la Universidad Católica Luis Amigó, las prácticas profesionales son de carácter semestral y se estructuran en tres niveles. Como parte de la preparación previa, los estudiantes asisten al curso Seminario de Práctica. En él, además de entender la naturaleza de las prácticas, deben realizar un ejercicio de observación y reflexión en terreno. Este primer acercamiento le permite al alumno dimensionar su nivel de preparación para esta etapa final de su formación. Entendemos en esta investigación, como alistamiento (readiness) para la práctica tanto la percepción de los participantes sobre si se sienten capaces de ejercer como trabajadores sociales y la preparación, así como las opiniones de los participantes sobre la manera como los aspectos de su programa educativo han facilitado su alistamiento (McSweeney \& Williams, 2019).

La práctica profesional del programa es una estrategia de enseñanza - aprendizaje que tiene lugar en un entorno de servicio. En el cual la relación recíproca entre la teoría y la práctica permite a los estudiantes desarrollar actividades acordes a las necesidades de las agencias y contextos donde realizan la práctica, así como evaluar las estrategias de intervención que promuevan el bienestar de los individuos, grupos y comunidades. Se denominan agencias las instituciones, organizaciones, empresas, fundaciones, etc. donde el estudiante realiza sus prácticas.

En el caso del programa de Trabajo Social, modalidad distancia, las prácticas se estructuran en tres niveles en forma de staff. Se entiende como staff un espacio de aprendizaje, acompañamiento y dirección donde el estudiante, en compañía del docente asesor, analiza los conocimientos y aplica las competencias profesionales en la Agencia, así como también reflexiona sobre su actuación y los resultados. Este espacio se organiza por área de intervención. Las áreas de desempeño en las que se insertan los estudiantes en 
Blanco-García

formación son: área familia, área socio educativa, área socio comunitaria, área organizacional, área salud y área calidad de vida, e innovación. Así, cada staff está presidido por un docente del área y los estudiantes vinculados asisten a dicho espacio. Si bien en las prácticas profesionales se vinculan otros actores como el coordinador de práctica o el cooperador en la agencia (jefe inmediato del estudiante en el lugar donde realiza las prácticas) y el estudiante también recibe instrucciones y asesoramiento y/o supervisión por parte de ellos, estos no hacen presencia en el staff.

El asesor, según el Manual de Prácticas, debe ser un docente activo vinculado contractualmente a la Universidad Católica Luis Amigó. Además, debe ser un profesional de las ciencias sociales y/o humanas con idoneidad e identificado con la misión institucional, con una formación académica óptima y comprobada experiencia en el ámbito de la intervención de la práctica del estudiante, con capacidad de trabajar de manera multidisciplinaria y en equipo.

Tiene bajo su responsabilidad orientar, acompañar y evaluar al estudiante en el ejercicio de su práctica, incluyendo el seguimiento al proyecto propuesto para la Agencia y al desempeño de sus funciones al interior de la Institución. Favorece el proceso de construcción teórico-práctica, que aporta a los estudiantes los elementos que permitan el logro de los objetivos planteados en los diferentes ámbitos de intervención de acuerdo con su experiencia.

Los asesores de prácticas realizan dos funciones: el acompañamiento y las visitas de seguimiento. En las asesorías semanales el docente asesor supervisa que la transferencia de aprendizaje del contexto formación al contexto de práctica corresponda a los objetivos y resultados acordados.

Según el Manual de Prácticas y Trabajo de Grado del programa, los objetivos esenciales del staff son:

- Desarrollar las habilidades y conocimientos de los estudiantes para evaluar y diagnosticar las problemáticas de la agencia.

- Vincular la teoría con la práctica

- Promover la reflexión sobre la Práctica Profesional

- $\quad$ Establecer objetivos y evaluar la efectividad de las acciones realizadas en la agencia.

- Asegurar el control de calidad de la práctica realizada.

- Fortalecer la toma de decisiones en casos de difícil resolución.

- Ayudar al practicante a ser más consciente de sus propias reacciones y respuestas a los usuarios del centro de práctica. 
- Permitir que el practicante socialice las situaciones desafiantes o amenazantes y que tienen efectos negativos en su proceso de práctica.

- Reflexionar sobre la identidad profesional a partir de las actividades desarrolladas en la agencia.

En cada uno de los niveles, el estudiante debe presentar varios productos en torno a la implementación de una intervención, además de cumplir las funciones asignadas por sus cooperadores en las distintas agencias. En el primer nivel se espera que el estudiante realice un diagnóstico social y de cuenta del mismo ante los actores de interés para luego, en el segundo nivel, plantear y ejecutar un proceso de intervención acorde a dicho análisis. En el tercer nivel, el practicante evaluará el ejercicio y socializará los resultados ante la comunidad académica y el cooperador de la Agencia. En todos los niveles el estudiante realiza una reflexión continua a través de su diario de campo y una reflexión final en la que se sintetiza y auto critica la experiencia.

Cuando el asesor exige que el estudiante justifique sus actividades en la Agencia, implica que éste analice las situaciones con rigor y aplique los conocimientos adquiridos (Puig-Cruells, 2020). En ese sentido, el proceso de aprendizaje autodirigido conlleva una continua recuperación de saberes para que el estudiante no solo aprenda a aprender, sino también aprender a hacer en el terreno. El aprendizaje autodirigido describe un proceso en el que los estudiantes toman la iniciativa, con o sin la ayuda de otros al diagnosticar sus necesidades de aprendizaje, formular sus metas, identificar los recursos humanos y materiales para el aprendizaje, elegir e implementar estrategias apropiadas, y evaluar los resultados de su proceso (Menz, Beer \& Bailey, 2019).

En el espacio del staff surgen unas inquietudes sobre la experiencia del estudiante en la modalidad: ¿Qué aspecto de su proceso de aprendizaje en la carrera le resultó más útil para enfrentar el proceso de práctica? ¿cómo recupera el estudiante estos saberes y autorregula dicho proceso? Si bien en la literatura se considera crucial el rol docente del tutor y supervisor de prácticas (Anabalón-Anabalón, Concha-Toro y Mora-Donoso, 2018; Escartín-Caparrós, Lillo-Beneyto, Mira-Grau, Suárez-Soto y Palomar-Villena, 2013; PuigCruells, 2020) y se abordan los aprendizajes en las prácticas, las experiencias de los estudiantes y la evaluación de las mismas (Concha-Toro, Anabalón-Anabalón, Lagos-San Martín y Mora-Donoso, 2020), la pregunta central de este artículo se centra en el proceso de aprendizaje autodirigido del estudiante en prácticas profesionales. 
Para presentar este análisis, el artículo, en un primer momento, da a conocer los aspectos metodológicos que guiaron el proceso investigativo realizado. Acto seguido, devela una reflexión sobre las experiencias de cinco estudiantes y cinco docentes entrevistados sobre sus experiencias en las agencias de práctica y actividades de enseñanzaaprendizaje en el staff. En un tercer momento, se plantean algunos retos metodológicos derivados de este análisis y reflexiones finales.

\section{Metodología}

Este artículo da cuenta de algunos de los resultados de la investigación "Preparación para la práctica profesional: el staff de prácticas como espacio de aprendizaje en el programa de Trabajo Social, modalidad distancia", realizada durante el año 2020. El interés en este espacio obedece a que el programa se oferta en esta modalidad desde 2016 y sus primeros estudiantes iniciaron el proceso de práctica en el año 2019.

Este estudio empleó una metodología cualitativa. Se realizaron entrevistas semiestructuradas con estudiantes y docentes asesores de prácticas del programa. En estas se exploraron las experiencias de los estudiantes en sus prácticas profesionales, cómo enfrentan las dificultades tanto en la Agencia como en el staff, sus percepciones sobre su alistamiento y preparación para este ejercicio, cómo recuperan los saberes previos y la evaluación preliminar de esta experiencia, entre otros temas de interés.

La entrevista es uno de los instrumentos más efectivos para obtener datos. Como señalan Gaitán-Moya y Piñuel-Raigada (1998):

[...] es el principal modo de recabar datos a partir de las declaraciones de personas que han vivido acontecimientos únicos o cuya documentación u opinión resulta irremplazable.

Resulta habitual su aplicación en los estudios de casos y en las investigaciones donde se pretende obtener una visión cualitativa de determinados aspectos de la vida de los sujetos, o de su forma de sentir, pensar o actuar en determinadas circunstancias, de los que nadie, sino ellos, pueden dar cuenta.

Desde el punto de vista de las estrategias de investigación, la entrevista cumple una función auxiliar, preparatoria o instrumental, por ejemplo, con el objeto de formular categorías. (p. 109)

Las entrevistas permitieron contrastar las percepciones de los investigadores con las de los sujetos entrevistados y, además, describir e interpretar aspectos del tema investigado que no se observan o se conocen a través de otras fuentes. En general, "las entrevistas se aplican, preferentemente, a personas que pertenecen al grupo de sujetos informados, es 
decir a aquellos que poseen un conocimiento acerca del referente investigado" (GaitánMoya y Piñuel-Raigada, 1998, p. 89).

En el momento de la realización de la investigación, el programa contaba con 17 estudiantes en prácticas profesionales y se encontraban en distintos ámbitos de intervención: seis en el primer nivel y once en el segundo. De esta población, accedieron a participar voluntariamente cinco estudiantes. Las agencias de práctica son variadas en términos geográficos y naturaleza misional. En cuanto a los docentes, su participación se basaba en el criterio de que debían ser asesores de práctica en uno de los ámbitos de intervención contemplados por el programa. Se realizaron cinco entrevistas a docentes que en el momento de la investigación cumplían con esta condición. Uno por cada área de intervención antes mencionadas, exceptuando el área de innovación.

Antes de su realización, el propósito fue explicado a cada uno de los participantes. Cada entrevista tuvo una duración promedio de una hora. Las propuestas para la investigación y diseño de instrumentos de recolección de información fueron aprobadas por el Comité de Ética de la Universidad Católica Luis Amigó. La participación de los estudiantes y docentes en el estudio fue voluntaria y todos los posibles participantes recibieron una invitación con información acerca del estudio. A todos se les dio tiempo para hacer preguntas y tomar una decisión informada sobre si deseaban participar. Quienes aceptaron participar completaron un formulario de consentimiento. La información general y propósito de la investigación se repitió en la etapa de entrevista.

Se les recordó a los estudiantes que participaron en las entrevistas que eran libres de retirarse en cualquier momento sin tener que dar una razón y sin perjuicio, además de que su participación no tenía ningún efecto sobre su rendimiento académico. Las entrevistas se grabaron en video y se transcribieron. Para proteger su confidencialidad, a los participantes se les asignó un código: EE\# para los estudiantes y ED\# para los docentes; todos los datos transcritos se anonimizaron.

Las limitaciones del proyecto se inscriben en el plano metodológico principalmente. La pequeña muestra de estudiantes obtenida, si bien no permite realizar generalizaciones, da insumos a los actores involucrados para hacer cambios significativos en el espacio y dinámicas de enseñanza-aprendizaje.

En el proceso de codificación de las entrevistas, apoyado en el programa Atlas.ti, la investigación integró elementos de los procedimientos deductivo e inductivo. Es decir, se partió de unas categorías iniciales basadas en el marco conceptual, las preguntas de investigación y, posteriormente, en la lectura y análisis del material para identificar temas emergentes. Las transcripciones se leyeron varias veces y se codificaron en relación con los juicios de los participantes sobre su experiencia en general en su práctica profesional. 


\section{Hallazgos}

\subsection{Percepciones sobre el alistamiento y preparación para las prácticas profesionales}

Los resultados en la literatura en cuanto a qué actividades específicas requieren los estudiantes son variados. Ya mencionamos que la relación supervisor y tutor son centrales. El docente asesor y el cooperador ofrecen modelos de buenas prácticas y elevan la conciencia del estudiante frente a su ejercicio (Fearnley, 2020). Otras actividades que están relacionadas con los estándares de ejercicio profesional necesitan ser priorizadas y son valoradas por los estudiantes (Roulston, Cleak, \& Vreugdenhil, 2018). La observación directa, el trabajo basado en casos, la retroalimentación informal y la autocrítica de los trabajos realizados también son algunos de los elementos identificados desde la perspectiva de los participantes en las investigaciones (Wilson \& Flanagan, 2019).

En cuanto a los resultados de la investigación, las percepciones generales sobre el nivel de alistamiento y preparación para las prácticas son mixtas. Existe temor y ansiedad por la elección del lugar de prácticas asociado al ámbito de intervención en el que se desempeñará el estudiante y las dinámicas organizacionales de la Agencia, sin embargo, hay reconocimiento de los conocimientos adquiridos en los cursos del programa. Así lo describe una de las participantes:

La verdad, digamos que sí, al principio asusta mucho [...] es decir, bueno, en algún momento lo pensamos y obviamente íbamos a llegar al tema de la práctica, pero sí de pronto uno ir revisando cómo podría abordar este momento el tema de la práctica, cómo se va sintiendo, cómo va digamos interiorizando muchos conceptos, todo lo que he visto de teoría cómo lo puedo ir entrelazando y cómo lo vas digamos a proyectar en esa práctica. [...] Además de lo teórico, yo siento que la universidad también [ha] ayudado mucho a que nosotros forjemos, en parte, ese carácter en que nosotros pensemos así digamos en algún momento no lo sintamos, pensemos y queramos esa mirada holística como todo el tema de poder articular el tema epistemológico, lo práctico, entonces ir ahí conectando de acuerdo con cada ejercicio de práctica. (EE1)

Llevar ese proceso con éxito en el contexto de las prácticas profesionales exige preguntarse cuál es el objetivo del ejercicio en la agencia, cuáles son las actividades más útiles y cómo implementar estrategias de supervisión. El estudiante no solo crea y ajusta una imagen previa de su alistamiento y preparación para la práctica, sino que también debe tener claras las realidades del ejercicio académico y práctico. La investigación de Joubert (2020), por ejemplo, indica que los estudiantes perciben el ejercicio de las prácticas como estresante y que demanda resiliencia, así como la ejecución de tareas fundamentales y la reflexión crítica. 
La respuesta anterior contrasta con la percepción de una de las docentes, quien identifica un bajo nivel de formación en términos de que, aunque los estudiantes cuentan con las herramientas necesarias, les cuesta comprender la lógica de su uso, especialmente cuando se implica una conexión de la realidad con el ejercicio académico (ED3). Esta dificultad, comenta, parece revelarse en el momento en el que el estudiante pasa de la lectura del material de apoyo a la aplicación y producción reflexiva. Así, el estudiante no logra conectarse con las actividades de trabajo profundo para materializar las propuestas. Adicional a esto, hay más manifestaciones y solicitudes por los asuntos logísticos, manejo del tiempo y seguimiento de instrucciones sencillas, sobre el dominio de las herramientas, uso de los conocimientos adquiridos, resolución de problemas a los que se enfrentan o hacia la práctica reflexiva.

Cabe resaltar que la selección de las agencias de práctica de los estudiantes está condicionada por las percepciones de dominio de los temas en un ámbito particular del Trabajo Social. En tres de los casos, los estudiantes escogieron como agencia su sitio de trabajo. Ello implica desde el Manual de prácticas la definición de tareas específicas asociadas al Trabajo Social. Una de las estudiantes explica:

Bueno es un momento en que se presenta ansiedad por lo desconocido, sin embargo, yo estoy haciendo las prácticas en lugar donde trabajo y quizás mi mayor preocupación era eso, los roles. Entonces yo soy secretaria y cómo voy a hacer para que me identifiquen como practicante, pero ha sido un proceso muy bonito los compañeros me han respetado ese espacio, me han dado ese lugar dentro el programa, ha sido muy significativo. (EE3)

En ese sentido, el estudiante identifica la necesidad de separar sus distintos roles dentro de la organización y empezar a cimentar la identidad como profesional del Trabajo Social. Si bien esta preocupación inicial del estudiante le exige un proceso de adaptación, en la perspectiva del docente asesor esto tiene sus ventajas: el practicante conoce la estructura y dinámica organizacional (ED1), así como los actores de la Agencia y los procesos administrativos que requiere su perfil (ED2). Este tipo de variables pueden considerarse como "obstáculos o como condición [es] de posibilidad" (Parola, 2020, p. 79).

Un aspecto en el que concuerdan ambos grupos de entrevistados es que los practicantes no comparten sus percepciones sobre su nivel de preparación y alistamiento para las prácticas entre ellos o con los docentes. Las respuestas recibidas sobre la pregunta ¿Has hablado con tus compañeros o asesores sobre tu percepción sobre tu nivel de preparación? son negativas. Las explicaciones dadas por los alumnos reflejan un entendimiento en común del reto que implica el ejercicio y que tales inquietudes se resuelven a "nivel personal". Esto puede repercutir en la percepción de distancia con el docente asesor y los demás participantes del staff: se trata de un ejercicio individual, 
Blanco-García

contrario a la idea misma de colaboración que la profesión vincula desde las ópticas constructivistas del saber y hacer.

En cuanto a la influencia de la modalidad en el nivel de preparación, los estudiantes manifiestan que los temores residen más en la percepción negativa que puedan tener los potenciales cooperadores sobre los procesos educativos a distancia apoyados por las tecnologías de la información y la comunicación, y la aparente falta de espacios prácticos en ellos. Es necesario anotar que la modalidad distancia del programa es entendida desde la perspectiva tradicional. Si bien el estudiante cuenta con un ambiente virtual de aprendizaje en el que encuentra los recursos de aprendizaje y espacios para los eventos evaluativos, los procesos de enseñanza y aprendizaje no son virtuales. Esta percepción puede afectar la selección de los estudiantes en los procesos de selección, pero no tanto las actividades que se realizan en el staff.

En cuanto a la modalidad, yo siento que no ha sido tan complejo como lo pensé, al inicio pensaba que iba tener mucha más dificultad para poder digamos acomodarme a la situación. [...] Este tipo de espacios ha derribado ese mito, sentir que hacerlo a distancia no funciona tanto como presencial. Entonces considero que sí es un buen espacio y que también va mucho en cómo las dos personas y el grupo del staff lo pueda abordar, cómo se pueda posesionar o empoderar de ese espacio, cómo se lo aprovecha al máximo, cómo se reciba de mejor manera las retroalimentaciones. (EE1)

De hecho, tres de las estudiantes manifiestan que la modalidad distancia apoyada por las TIC supone una posibilidad de desarrollo de habilidades. Una de ellas, por ejemplo, expresa:

[...] digamos que la modalidad distancia me ha dado la posibilidad de manejar las TICS. [...] en esta modalidad le toca a uno investigar, innovar. Sí, y entonces digamos del proceso de la pandemia me ha tocado obviamente rebuscarme [...], pienso que es un punto a favor en cuanto a este proceso de la modalidad distancia. (EE4)

\subsection{Utilidad percibida de las actividades de aprendizaje en el staff de prácticas}

A las preguntas relacionadas con la utilidad de las distintas actividades del staff de prácticas para el apoyo del aprendizaje autodirigido, los estudiantes confirman la importancia del diario de campo como dispositivo de memoria. Su gran aporte es la recuperación de saberes previos mediante las notas de clase y los recursos disponibles en el ambiente virtual de aprendizaje. 
Lo que hago es retomar y muchas veces, llegar a la casa, volver a esos cursos anteriores a mirar un trabajo, a mirar un documento, que es el que me habla de esas diferentes formas de intervención, como lo que tengo que hacer. [...] he sentido últimamente, la base de datos es muy importante, [...] que es un recurso que la universidad paga, pero cuando son lecturas en línea o cuando son préstamos, me estoy quedando sin el insumo, no tengo en qué apoyarme. Si tomo nota de cómo se llama el documento tengo que buscarlo, el documento como tal no me queda. Yo he sentido como la ausencia del documento que quizás, que en cursos anteriores yo me apoyaba, ahora últimamente como se utiliza la base de datos y todo eso, ese insumo ya no lo tengo. (EE2)

Otra de las actividades más valiosas han sido la presentación de avances y resultados y la retroalimentación a los diarios de campo mencionados. Una de las docentes explica:

[...] primero es preguntarles cómo les fue esa semana, esas dos semanas, ¿qué actividades realizaron? ¿qué cosas nuevas surgieron? ¿cómo les fue allí? En especial, me interesa saber ¿qué actividades están realizando?, al conocer esto yo puedo ir enganchando las devoluciones y en especial el discurso a lo que ellas hacen allá y también monitoreando qué es lo que las ponen a hacer. (ED2)

Las actividades que el estudiante encontrará más útiles serán aquellas que le permitan reforzar continuamente lo que sabe y lo que sabe hacer de manera recursiva y ajustar esas percepciones. La categorización que nos presenta Carl Newport (2017) es útil para entender este desafío. Muchas de las actividades que realiza el estudiante en la práctica y en su vida académica son superficiales; tareas que desde el punto de vista cognitivo no son exigentes puesto que caen en el orden de lo logístico. Si bien reconocemos que son importantes para adquirir ciertas habilidades de adaptación, no lo serán tanto a largo plazo. Muchas de ellas son asignadas por el cooperador y se corre el riesgo de que roben el protagonismo en el día a día del practicante.

Por el otro lado, las tareas que permiten el trabajo a fondo, como lo denomina el autor y que son ideales para el aprendizaje, son aquellas "que se llevan a cabo en un estado de concentración desprovisto de distracciones, de tal manera que las capacidades cognitivas llegan a su límite máximo" (Newport, 2017, p. 13). Esto, explica el autor, requiere de dos aptitudes esenciales: el dominio rápido de los conocimientos difíciles y la capacidad para producir en un nivel superior, en términos de calidad y velocidad. Es allí donde consideramos que el staff de prácticas profesionales, además de las tareas de asesoramiento y supervisión, debe ofrecer la oportunidad para el trabajo a fondo que necesita el estudiante. Sin embargo, para Ahrens (2020) hay diferencias en el tipo de atención que se requiere: "La atención centrada es diferente de la atención sostenida, la cual necesitamos para mantenernos centrados en una tarea durante un periodo de tiempo más largo y es necesaria para aprender, comprender o llevar a cabo algo" (p. 71). 
Blanco-García

El practicante de Trabajo Social aprende haciendo y aprende viendo para hacerlo mejor. Stiglitz y Greenwald (2015) explican que "si experimentamos conscientemente con el trabajo, buscando formas alternativas de hacer lo que hacemos, es probable que aprendamos más que si esperamos un momento de genialidad en el que tengamos una brillante revelación sobre una forma alternativa de hacer lo que hemos estado haciendo" (pp. 94-95).

Además de los conocimientos básicos y profesionales, el estudiante deberá desarrollar sus habilidades de concentración, diligencia, recuperación de saberes previos, comunicación, retención, intuición y experimentación. Este listado de habilidades no es exhaustivo, pero da un panorama general de las mismas. Un listado más detallado podría plantearse, como lo argumenta Menz, Beer, y Bailey (2019), desde una categorización en tres frentes: cognitivo, intrapersonal e interpersonal. Finalmente, otros autores enfatizan que deben realizarse cambios en los programas académicos de Trabajo Social para integrar el desarrollo de la identidad profesional en los materiales y actividades de aprendizaje (Moorhead, Bell, Jones-Mutton, Boetto, \& Bailey, 2019).

De nuevo, el factor logístico juega un papel negativo en el desarrollo de las actividades; se trata, como lo expresa Newport (2017), de una inclinación por las tareas superficiales en detrimento de la producción en términos de profundidad. Los docentes asesores concuerdan que el nivel de habilidades en actividades profundas requiere mucho más tiempo del planeado y que deberían ser asuntos dominados, puesto que son elementos transversales a la labor del trabajador social vistos en distintos cursos. El planteamiento de los diagnósticos o el diseño de indicadores son ejemplos de estos asuntos. Es cierto que, por definición, estas actividades requieren un esfuerzo mayor, pero la retroalimentación y superación de las dificultades en estos casos se torna repetitiva. Esto se agrava por el no seguimiento de algunas instrucciones dadas. En ese sentido, los resultados se alinean con lo expresado por Bogo (2006): las actividades de aprendizaje reflexivas y conceptuales, así como las que fomentan la autocrítica de los estudiantes son las mejor valoradas desde la perspectiva estudiantil.

De otro lado, las estudiantes en el nivel tres de práctica refieren que preferirían que el acompañamiento fuera más individualizado o que el número de alumnos en cada staff fuese más reducido.

Yo estaba muy acostumbrada a que, digamos, ese staff ha sido muy personalizado porque yo era la única practicante que estaba ahí con la profe. Entonces era más ese contacto con ella y yo era la que me sentaba ahí y hablamos de esto y esto. [...] al entrar otro estudiante a la práctica limita como esa conexión, ese staff de práctica inicialmente era ella y yo. Como algo muy egoísta, pero sí era muy enfocado a lo que yo le decía. En cambio, ahora es tu qué opinas, el otro qué opina y entonces empiezan hablar aquí y empieza hablar acá, digamos es un poco más disperso. (EE4) 


\subsection{Aprendizaje autodirigido}

Desde las respuestas obtenidas en las entrevistas se pueden identificar distintos niveles de apropiación del aprendizaje autodirigido de acuerdo al nivel de prácticas de los estudiantes. La autorregulación de las actividades está mediada por la percepción de preparación para la práctica y los resultados de las actividades del staff.

Yo pienso que, obviamente, cuando uno no estaba haciendo la práctica, cumplir con los trabajos de la universidad... pero ya en el proceso de práctica si uno ve como la necesidad de trabajar en ciertos aspectos pues han llevado a ese trabajo autónomo de estar como a la par, digamos, con las cooperantes o con las situaciones actuales. (EE4)

En las primeras etapas de las actividades los estudiantes reciben una retroalimentación externa objetiva que impacta su imagen de nivel de alistamiento. En estas tareas que requieren la recuperación de saberes, parece ocurrir el efecto de mera exposición: si el estudiante lee algo o sabe que tiene disponible la información que requiere la tarea no significa que la domine, es decir, confunde familiaridad con habilidad. Este efecto parece venir desde tiempo atrás.

En el trabajo de apoyo al estudiante se pueden presentar diferencias muy marcadas entre asesor y cooperador coherente con las funciones descritas por Puig-Cruells (2020). Estas funciones darían paso a modificaciones diversas al nivel de preparación. Una de las estudiantes describe la situación de la siguiente forma:

Sí, con respecto a la cooperante es de más haga esto y de tareas muy específicas, mientras que el staff de práctica le da posibilidad de dar respuesta a tareas específicas de diferentes herramientas con otros actores, desde el diálogo con la misma docente. Mientras que la cooperante es especifica: "necesito que trabajemos este tema", en el staff de práctica "cómo podemos trabajar este tema, que sí les llegue a los estudiantes". (EE4)

Uno de los docentes explica:

[...] en cuanto al proceso, es un proceso de corresponsabilidad y en algún momento le decía al estudiante, depende de usted el proceso académico, cierto, yo simplemente voy a acompañar en este proceso no voy a hacer el trabajo la voy a orientar así que, el resultado del trabajo va ser casi el $80 \%$ de lo que usted empiece a construir desde el acompañamiento que yo le estoy dando. (ED1)

Cuando el estudiante entra a trabajar a partir de las devoluciones, se exige de él la lectura crítica del trabajo. Ahrens (2020) hace una distinción importante de este paso: "Aunque asumir el papel de crítico no sea lo mismo que ser un lector imparcial, es suficiente para detectar la mayor parte de lo que se nos ha escapado" (p. 72). 
[...] algo que considero que ayuda muchísimo es la retroalimentación de los diarios de campo. Ese ha sido digamos uno de los factores que en lo personal me han servido mucho para entonces encontrar ese norte en cuanto si es por este lado. Entonces necesitas el tema epistemológico que puedas sacar una conclusión mucho más acorde a lo que se está exigiendo, que puedas exigirte si en algún momento no se ha dado el mejor resultado. Y digamos ya la presentación oficial o la que se pueda dar para el cierre de práctica, porque, de acuerdo a todas esas exigencias, a todas esas correcciones, ir consolidando ese producto que vas a entregar después, ese proceso que llevas en cada staff y podérselo presentar a tu cooperante acompañado de ese profesor que te está guiando ha sido también muy importante. (EE1)

\section{Conclusiones}

Este artículo se ha enfocado en tres elementos de las prácticas profesionales en Trabajo Social dinamizadas desde un espacio tipo staff, con la esperanza de que, al comprender su influencia en el aprendizaje autodirigido de los estudiantes, podamos tener más éxito a la hora de diseñar y dinamizar las actividades de enseñanza-aprendizaje que se requieren.

En ese sentido, las prácticas profesionales y la construcción de la identidad del estudiante como trabajador social pasan por la autorregulación de su rol como estudiante y el autodiagnóstico de su alistamiento y preparación para estos ejercicios académicos. Proceso que debe darse teniendo en cuenta y diferenciando la utilidad de las retroalimentaciones dadas por el asesor y el cooperador.

Sin embargo, la imagen que va construyendo el estudiante sobre su nivel de alistamiento y preparación a las prácticas requiere, durante los semestres anteriores, de ejercicios reflexivos para que se ajuste de manera apropiada y puedan tomarse acciones constructivas.

Existen preguntas que deben incluirse en estos diseños. En particular, ¿cómo incentivar el trabajo profundo de los estudiantes desde etapas previas? ¿cómo ayudarlos a desarrollar las habilidades para que las actividades más superficiales no choquen con las que requieren una práctica enlazada estrechamente con la teoría? Estos asuntos no son exclusivos de la educación presencial. Los resultados de la investigación aquí mencionados apoyan este punto. La modalidad distancia puede y debe generar los espacios de trabajo profundo que requiere el éxito de los estudiantes en sus prácticas profesionales y en la formación de su identidad profesional.

Para finalizar, las agencias de práctica también cumplen un papel importante al entender que las prácticas no pueden dejar de privilegiar la reflexión y la formación en favor de hacer muchas cosas superficiales como señal de productividad. 


\section{Referencias bibliográficas}

Ahrens, S. (2020). El método Zettelkasten: Cómo tomar notas de forma eficaz para impulsar la escritura y el aprendizaje de estudiantes, académicos y escritores de no ficción (G. Carmona, Trad.). Estados Unidos: Independently published. (Obra original publicada en 2017).

Anabalón-Anabalón, Y., Concha-Toro, M., y Mora-Donoso, M. (2018). Tutorías académicas y Prácticas Profesionales: invitación al desempeño profesional inicial en la formación trabajadores sociales. Revista UCMaule, (54), 51-64. doi: 10.29035/ucmaule.54.51.

Bogo, M. (2006). Field Instruction in Social Work: A Review of the Research Literature. The Clinical Supervisor, 24(1-2), 163-193. doi: 10.1300/J001v24n01_09.

Concha-Toro, M. del C., Anabalón-Anabalón, Y. B., Lagos-San Martín, N. G., y MoraDonoso, M. L. (2020). Prácticas profesionales y Trabajo Social. Una revisión de la literatura en educación superior. Pensamiento Educativo: Revista de Investigación Educacional Latinoamericana, 57(1), 1-19. doi: 10.7764/PEL.57.1.2020.5.

Escartín-Caparrós, M. J., Lillo-Beneyto, A., Mira-Grau, F. J., Suárez-Soto, E., y PalomarVillena, M. (2013). El proceso de supervisión en las prácticas de trabajo social. En M. T. Tortosa Ybáñez, J. D. Álvarez Teruel y N. P. Buades (Coords.), XI Jornadas de Redes de Investigación en Docencia Universitaria. Retos de futuro en la enseñanza superior: docencia e investigación para alcanzar la excelencia académica (pp. 679-691). Alicante: Universidad de Alicante. Vicerrectorado de Estudios, Formación y Calidad. Recuperado de http:/ / rua.ua.es/dspace/handle/10045/31305.

Fearnley, B. (2020). Enhancing social work student learning: Converging Bronfenbrenner, Bourdieu and practice learning. Social Work Education, 39(2), 214-226. doi: 10.1080/02615479.2019.1618258.

Gaitán-Moya, J. A., y Piñuel-Raigada, J. L. (1998). Técnicas de investigación en comunicación social: elaboración y registro de datos. Madrid: Síntesis.

García-Barreto, J. I., Zambrano-Cárdenas, A. E., y Martínez-Suárez, M. (2017). Reflexión documental de las prácticas profesionales de trabajo social en Colombia. Perspectivas, (8), 60-68. Recuperado de https:/ / revistas.uniminuto.edu/index.php/Pers/article/view/1613.

Joubert, M. (2020). Social work students' perceptions of their readiness for practice and to practise. Social Work Education. doi: 10.1080/02615479.2020.1749587.

McSweeney, F., \& Williams, D. (2019). Social care graduates' judgements of their readiness and preparedness for practice. Social Work Education, 38(3), 359-376. doi: 10.1080/02615479.2018.1521792.

Menz, E., Beer, J. de, \& Bailey, R. (Eds.). (2019). Self-directed learning for the 21st century: Implications for higher education. Recuperado de https: / / www.doabooks.org/doab?func=fulltext\&uiLanguage=en\&rid $=45286$.

Moorhead, B., Bell, K., Jones-Mutton, T., Boetto, H., \& Bailey, R. (2019). Preparation for practice: Embedding the development of professional identity within social work curriculum. Social Work Education, 38(8), 1-13. doi: 10.1080/02615479.2019.1595570. 
Blanco-García

Newport, C. (2017). Enfócate. Consejos para alcanzar el éxito en un mundo disperso. España: Paidós.

Parola, R. N. (2020). Problematizando las Prácticas preprofesionales en Trabajo Social. Desafíos y perspectivas. Prospectiva. Revista de Trabajo Social e Intervención Social, (29), 73-88. doi: 10.25100/prts.v0i29.8714.

Puig-Cruells, C. (2020). El rol docente del tutor y supervisor de prácticas en Trabajo Social: Construcción de la reflexividad y el compromiso durante la formación a través de la supervisión. Prospectiva. Revista de Trabajo Social e Intervención Social, (29), 57-72. doi: $10.25100 /$ prts.v0i29.8084.

Roulston, A., Cleak, H., \& Vreugdenhil, A. (2018). Promoting Readiness to Practice: Which Learning Activities Promote Competence and Professional Identity for Student Social Workers During Practice Learning? Journal of Social Work Education, 54(2), 364-378. doi: 10.1080/10437797.2017.1336140.

Stiglitz, J. E., y Greenwald, B. C. (2015). La creación de una sociedad del aprendizaje.: un nuevo enfoque hacia el crecimiento, el desarrollo y el progreso social: conceptos básicos análisis. España: Grupo Planeta.

Wilson, E., \& Flanagan, N. (2019). What tools facilitate learning on placement? Findings of a social work student-to-student research study. Social Work Education, 40(2), 1-17. doi: 10.1080/02615479.2019.1702636. 


\section{OTROS ARTÍCULOS DE PROSPECTIVA No. 32 DE 2021}

\section{EDITORIAL}

Compromisos, dilemas y desafíos del Trabajo Social con dimensión colectiva en tiempos de pandemia

Enrique Pastor-Seller

\section{ARTÍCULOS}

La narrativa biográfica como alternativa para la comprensión del Conflicto y la violencia en Colombia: una experiencia pedagógica universitaria

Sara Sofía Castaño-Barco y Claudia BermúdezPeña

Experiencias de transición a la vida adulta de jóvenes que migraron solos. Un estudio en Aragón (España) Daniel Jiménez-Franco; Raquel Berzosa-Callén y Chabier Gimeno-Monterde

Fundamentals of Education in Intervention with Young Offenders. A Critical Review of a 'Successful' Case in Andalucía, España

David Herrera-Pastor

Subjetividad juvenil: lecturas desde y para el Trabajo Social en Colombia

Daniela Joya-Valbuena

Participación politica de los jóvenes del Valle del Cauca, Colombia, en la toma de decisiones públicas

Luis Eduardo Gutiérrez-Rojas y Amanda Ramírez-Giraldo

Victimización y perpetración de violencia en pareja adolescente y redes de apoyo en Colombia. Análisis con perspectiva de género

Johanna Alexandra Reina-Barreto
Apoyo social percibido, autoestima y maternidad adolescente: entre el respeto y la intrusión. Estudio en Traiguén, Chile

Gloria Mora-Guerrero, Luisa Escárate-Colín, Carol Espinoza-Lerdón y Andrea Peña-Paredes

El desarrollo en el Trabajo Social de Colombia: un campo transversal, disperso y polivalente

Maira Judith Contreras-Santos

Análisis de la situación laboral de las alimentadoras en la caficultura de la zona central colombiana, a la luz de la teoría de Nancy Fraser

Pablo Andrés Arango-Giraldo

Prioridades de investigación en la Obra social de empleados públicos, Mendoza, Argentina. Un estudio Delphi sobre producción de conocimientos en la seguridad social

Cecilia Amalia Molina

Producción de conocimiento y toma de decisiones. Relaciones entre academia y política pública para las familias en Colombia

José Raúl-Ruíz y Sandro Leonardo MunévarVargas

El staff de prácticas profesionales en Trabajo Social: espacio de fomento del aprendizaje autodirigido. Un estudio de caso en la Universidad Católica Luis Amigó, Colombia

Cristian Eduardo Blanco-García

\section{ARTISTA INVITADO}

Margie Reinel-Aguilar Ao Cicadidae

Prospectiva

\section{PROSPECTIVA Revista de Trabajo Social e Intervención Social}

No. 32 • jul.-dic. 2021

e-ISSN: 2389-993X • Universidad del Valle 